\title{
From Passive Tool Holders to Microsurgeons: Safer, Smaller, Smarter Surgical Robots
}

\author{
Christos Bergeles, Member, IEEE, and Guang-Zhong Yang, Fellow, IEEE
}

\begin{abstract}
Within only a few decades from its initial introduction, the field of surgical robotics has evolved into a dynamic and rapidly growing research area with increasing clinical uptake worldwide. Initially introduced for stereotaxic neurosurgery, surgical robots are now involved in an increasing number of procedures, demonstrating their practical clinical potential whilst propelling further advances in surgical innovations. Emerging platforms are also able to perform complex interventions through only a single entry incision, and navigate through natural anatomical pathways in a tethered or wireless fashion. New devices facilitate superhuman dexterity and enable the performance of surgical steps that are otherwise impossible. They also allow seamless integration of micro-imaging techniques at the cellular level, significantly expanding the capabilities of surgeons. This paper provides an overview of the significant achievements in surgical robotics and identifies the current trends and future research directions of the field in making surgical robots safer, smaller, and smarter.
\end{abstract}

Index Terms-Minimally invasive surgery, surgical robots, microrobots.

\section{INTRODUCTION}

Five decades ago, Driller and Neumann published their electromagnetic biopsy device [1], the first medical robot related paper of this journal. It took just half a decade for the still clinically-unrealised concept of electromagnetic cerebral catheter steering to be presented by Askenasy et al. [2], and the concept of superconductive intravascular magnetic navigation to be proposed by Montgomery et al. [3]. Although the Food and Drug Administration (FDA, USA) had undertaken regulatory measures for the translation of medical devices since 1976, the concept of Health and Service Robotics had not been introduced until 1984, when the United States Congress was urged to support "innovative research in functional rehabilitation of cognitive capabilities, speech, mobility, and manipulation" [4]. During that period, robots in medicine were considered mainly as rehabilitation devices and nurse assistants [5].

It was not until 1988 that the experimental evaluation of stereotaxic needle insertion using the first surgical robot, a conventional industrial Unimation PUMA 200, was published by Kwoh et al. [11] in T-BME. This publication heralded the explosion of research on surgical robots and their gradual introduction to operating theatres, the first clinically evaluated

Manuscript received April 8, 2014.

C. Bergeles and G.-Z. Yang are with the Hamlyn Centre, Imperial College London, SW7 2AZ, U.K.. Correspondence: c.bergeles [at] imperial.ac.uk.

Copyright (c) 2013 IEEE. Personal use of this material is permitted. However, permission to use this material for any other purposes must be obtained from the IEEE by sending an email to pubs-permissions@ieee.org.

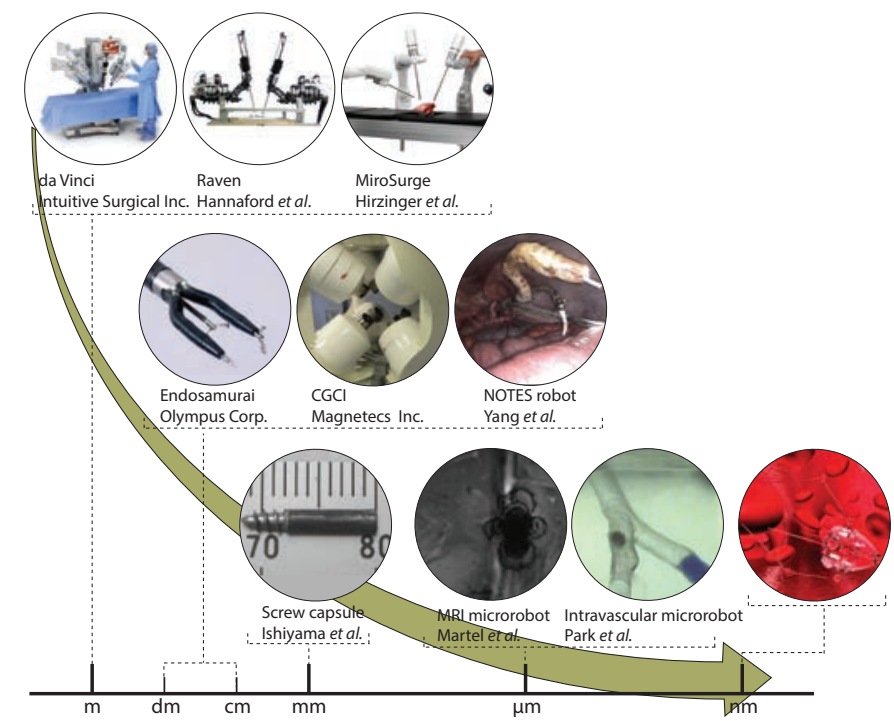

Fig. 1. Exemplary surgical robots along different size scales. "da Vinci" image: (c) 2013 Intuitive Surgical, Inc.; "Raven" image: (c) Applied Dexterity, Inc.; "MiroSurge" image: (c) DRL; "Endosamurai", adapted from [6], (c) 2010 Baishideng; "CGCI", (c) Magnetecs, Inc; "NOTES robot", "Screw capsule", and "MRI microrobot" images: reprinted with IEEE permission from [7], [8], [9]; "Intravascular microrobot": reprinted with Elsevier permission from [10]; Illustration of nanorobot: (C) IEEE.

robots being "ROBODOC" [12], a robot for precise hip replacement, developed by Taylor et al. [13], and "PROBOT", a robot for prostatectomy, developed by Davies et al. [14]. Since then, surgical robots have advanced significantly, becoming safer, more acceptable, and more versatile, resulting in a multi-billion industry led by the exemplary success story of "da Vinci" from Intuitive Surgical Inc. [15]. From the macro-scale surgical robots that originated from industrial manipulators, the field is also progressing towards smarter and smaller systems, with exciting research conducted on millimetre- and even micrometre-scale devices (see Fig. 1). Nowadays, microsurgical platforms are being developed for virtually all parts of the human anatomy (see Fig. 2).

Science fiction, however, had long before included robotic surgeons in its themes, with movies portraying both intracorporeal navigation of microrobots (Fantastic Voyage, 1966) and robotic surgery (The Empire Strikes Back, 1980). Several reasons for this relative delay have been identified, with the depiction of robots as unwanted (Metropolis, 1920) and unsafe (Rossum's Universal Robots, 1923) being important factors. Robots in the operating theatre were characterised as a potential "bull in a china shop", despite their precision 
being superior to the surgeon's [16]. Only in 1991 did safety guidelines for "taming the bull" appear to assist in surgical robot development [17].

With the rapid advance of surgical robotics in recent years, it is timely to provide an overview of the field's progression since the first surgical robots were introduced. We do not attempt to perform an exhaustive taxonomy of research publications. The long list of high-quality publications and products makes inadvertent omission inevitable, thus, we refer the readers to existing reviews on past and state-of-the-art surgical robotics [18]-[28]. In this paper, we focus on representative publications and robotic systems that helped shape this research field and identify open research directions and socioeconomic issues. When possible, we highlight systems evaluated in vivo. Representatives are shown in Table I.

Building on the classification of Salisbury et al. [21] and Rebello [22], we focus on different generations of robotic surgery. Thus, Section II describes the transition from industrial manipulators to untethered intracorporeal microsurgeons. Ideas on the field's progression and outstanding challenges are discussed in Sec. III, and Sec. IV presents the conclusions.

\section{Surgical Robots: Past And Present}

This section follows the evolution of surgical robots. From one generation to the next, invasiveness and collateral tissue damage is reduced while surgical dexterity is augmented. Laparoscopic surgery, considered as generation zero, is the starting point in Sec. II-A. Subsequently, Sec. II-B discusses stereotaxic robotic surgery and needle insertion, i.e., firstgeneration systems. The second-generation systems for dextrous minimally-invasive robotic surgery are the focus of Sec. II-C. Systems for natural-pathway navigation are thirdgeneration systems discussed in Sec. II-D, and naturally lead to the untethered milli- and micro-robotic surgical systems of Sec. II-E. Each generation identified shortcomings of existing procedures and characteristics that surgical robots should exhibit. These characteristics were implemented by subsequent systems to deliver treatments of higher versatility, safety, and success. Each generation contains smaller systems that are easier to introduce in the surgical workflow, thus demonstrating the trends of miniaturisation and increased dexterity and accessibility.

\section{A. Laparoscopy - $0^{\text {th }}$ Generation}

In laparoscopy (also termed minimally invasive surgery (MIS) or keyhole surgery), access to the pathology location is gained by inserting and manipulating elongated surgical tools through a small number of abdominal incisions. For better visualisation of the operating site and increased workspace, the abdomen is inflated with carbon dioxide. Even though robots were absent from initial MIS endeavours, it was engineering developments on endoscopes and surgical tools that enabled the adoption of these new techniques, starting with the first laparoscopic cholecystectomy by Mühe in 1985 [29].

The advent of laparoscopic practices marks the transition from traditional open-surgery to MIS techniques. Their wide adoption is due to improved patient recovery times, reduced trauma, and reduced hospitalisation costs [30]. Despite these advantages, MIS faces significant hurdles complicating its application particularly to the newer surgical domains of single-port access, intraluminal and transluminal surgery [28].

These complications revolve around ergonomic factors associated with limited sensory feedback of surgeons, i.e., misalignment of visuomotor axes, and a high demand on manual dexterity required to manipulate the laparoscopic instruments. More specifically, since most endoscopes provide 2D images, the surgeons operate while looking at a screen. Stereoscopic depth perception is commonly absent and their visual estimation skills are impaired [31]. Haptic feedback is also limited as they have to manipulate the surgical tools while tackling the fulcrum effect resulting from the constraining incision ports [32]. The lack of feedback makes certain types of microsurgery particularly challenging. Retinal microsurgery, for example, requires forces that are at the limits of human perception [33], rendering safe tissue manipulation highly demanding.

Surgical robotic systems, operating either autonomously or in a teleoperated fashion, were proposed as clinical solutions to the problems of precise manipulation, visualisation, and force feedback. The introduction of these systems marks the start of the first generation of robotic surgery.

\section{B. Stereotaxic Robotic Systems - $1^{\text {st }}$ Generation}

The first generation of surgical robots relates to stereotaxic interventions in neurosurgery and orthopaedics. These systems focus on increasing surgical accuracy rather than returning sight or haptic sensation to the surgeon, thus primarily dealing with the lack of dexterity in MIS. Their pioneering ideas are the foundation of state-of-the-art robotic surgical systems.

The first application of a robot in surgery was performed by Kwoh et al. [11]. The industrial PUMA 200 manipulator was registered to a stereotaxic frame, was positioned to define the entry orientation and location of a surgical needle, and was subsequently switched off. The surgeon proceeded in manually advancing the needle through the corridor defined by the robot, achieving placement accuracy below $1 \mathrm{~mm}$. Kwoh et al. subsequently improved their approach to remove the first intracranial tumour [40]. This innovative use of industrial robots evolved into the first neurosurgical robot, "Neuromate" [41], which has been granted FDA approval and is now commercialised by Renishaw Plc. Since "Neuromate" and subsequent similar approaches [42] initially defined a surgical entry path, they are considered as passive tool holders [19].

The underlying trend in stereotaxic neurosurgical robots gradually steered away from industrial manipulators towards developing dedicated systems. "Minerva" is an example of a mechatronic system that was designed to satisfy sterilisation constraints, the requirements for dynamic updates through intraoperative CT-based visualisation, and established safety requirements [43]. More recent smaller-scale systems, such as "NeuroArm" from Sutherland et al. [44], highlight the clinical demand on smaller and less intimidating systems that can be integrated easily with existing surgical flow into the operating room. Newer state-of-the-art needle-insertion robots push the boundaries of miniaturisation by being skull- and 


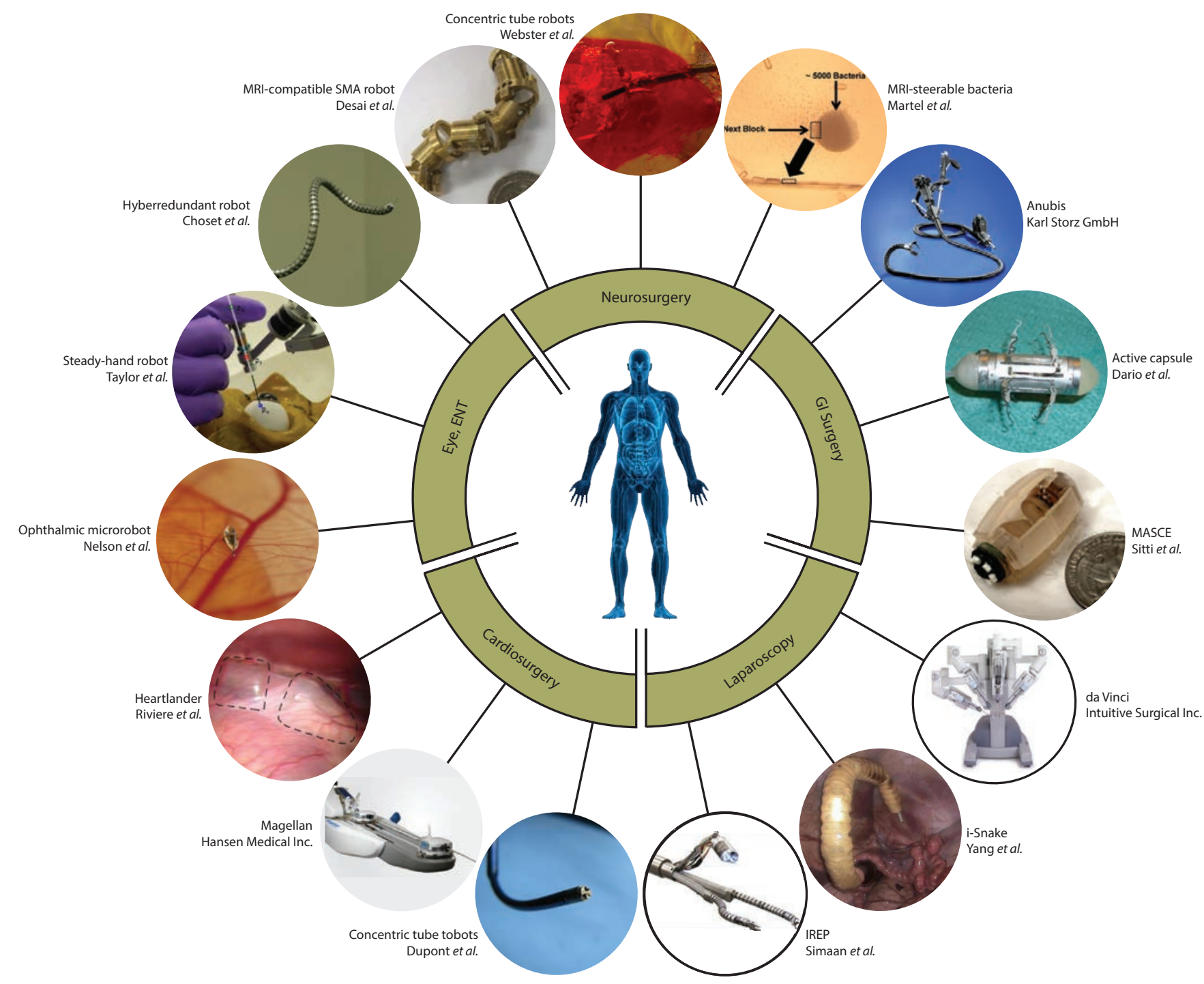

Fig. 2. Exemplary surgical robots for different applications.

"Ophthalmic microrobot": (c) ETH Zurich; "Steady-hand robot": adapted from [34], (c) SAGE publications; "Hyperredundant robot", (c) Carnegie Mellon; "MRI-compatible SMA robot": reprinted with IEEE permission from [35]; "Concentric tube robots" (top): (c) Vanderbilt University; "MRI-steerable bacteria": reprinted with IEEE permission from [36]; "Anubis": (c) Karl Storz GmbH; "Active capsule": (c) Scuola Superiore San't Anna; "MASCE": reprinted with IEEE permission from [37]; "da Vinci": (C) 2013 Intuitive Surgical Inc; "i-Snake": reprinted with Springer permission from [38]; "IREP": (c) Vanderbilt University; "Concentric tube robots" (bottom): (c) Boston Children's Hospital; "Magellan": (c) Hansen Medical Inc.; "Heartlander": reprinted with IEEE permission from [39]. Representation of systems inspired by [25] by Nelson et al.

spine-mountable [45], [46], and by expanding their application area to cover kidney, prostate and lung procedures [47].

Towards improving accuracy, newer stereotaxic systems integrate non-ionising imaging modalities, such as Magnetic Resonance Imaging (MRI). Hence, both accurate tool manipulation and harmless intraoperative visualisation are possible. The first guidelines for the design of MRI-compatible robotic mechanisms were published by Jolesz et al. in 1999, with Kikinis et al. employing them to design the first MRI-compatible robot for neurosurgery [48]. A clinically-approved system for MRI-compatible needle insertion is the "Innomotion" arm (Synthes Inc.) [49]. This pioneering research presented ideas that motivated further endeavours on MRI-compatible system development. Examples include the MRI-compatible prostate biopsy systems developed by Whitcomb et al. [50] and Fichtinger and Iordachita et al. [51], and even MRI-powered needle-insertion robots by Dupont et al. [52]. Still, these systems essentially perform needle orientation, positioning, and insertion, and are hence classified as first generation.

Orthopaedic surgical robots evolved in parallel to neurosurgical systems. "ROBODOC", the first commercially successful surgical robot [12], was developed by Taylor and Kazanzides et al. in IBM and commercialised by Integrated Surgical Systems Inc. "ROBODOC", clinically evaluated in 1992, performed total hip replacement. It was initially based on a modified SCARA robot, and its operation involved acquiring a CT scan of the patient anatomy and subsequently developing a surgical plan to mill the location that receives 
the hip replacement implant. The surgeon was supervising the execution of the plan and intervening for cancellation or adaptations as necessary. The system implemented six-axis force sensing for safety, acknowledging the importance of force-feedback in surgery [13].

"ROBODOC" was fully autonomous, but a competing system, "AcroBot" from Davies et al., operated synergistically with the surgeon [53]. "AcroBot", i.e., active constraints robot, introduced a new telemanipulation concept wherein the surgeon was actively guiding the surgical tool to perform knee machining for implant reception. Instead of blindly following the surgeon's motion, active constraints ensured that the robot operates only within a predefined allowable region. The notion of telemanipulation using active constraints is an integral component of many state-of-the-art surgical robots, especially in domains that require micrometre accuracy [34], [54], [55].

Similar to neurosurgical needle-insertion robots, orthopaedic robots are evolving towards smaller devices that fit in the operating theatre with ease. One such example is "iBlock" (Praxim Inc.), a total-knee arthroplasty robot. "iBlock" mounts directly to the bone, thus preserving the registration between itself and the anatomy [27], [56].

It is not surprising that the first robotic systems were applied to neurosurgery and orthopaedic surgery, as both are characterised by operation within well defined bone structures, i.e., the skull, and the hip or knee. This allows robots to be registered to the anatomy using bone-mounted landmarks whose position changes little during surgery. Hence, the established anatomy/robot registration can be maintained throughout the procedure without adaptation to the robotic mechanism.

The developed systems allow interventions of increased accuracy, and their success supported the acceptance of robots operating in surgical suites. Through iterations and building on existing research contributions, systems gradually became more lightweight and compact. They are, however, not designed to allow visualisation, haptic feedback, or surgical dexterity in multiple degrees of freedom. These issues are a requirement for improving the outcome of minimally invasive interventions that involve tissue manipulation. This is the focus of the next generation of robotic systems, which are based on the integration of imaging modalities, the notion of synergistically operating with the surgeon, and the now established limitations of surgical and robotic capabilities.

\section{Rigid Dextrous Robots for MIS - $2^{\text {nd }}$ Generation}

Initial success in stereotaxic interventions and needle manipulation demonstrated the potential of introducing robots in the operating theatre. With the increasing acceptance of laparoscopic MIS, there was higher demand for superhuman dexterity and visualisation via robotic assistance. The robots of this generation approach the problem of operating in confined body cavities and dextrously manipulating tissue. They were initially envisioned as endoscope manipulators meant to relieve surgical assistants. Indeed, their first field of application in laparoscopy was manipulation of the imaging system via tracking of surgical tools [57] or surgeon head motion [58].

The first robotic soft-tissue surgeon was "PROBOT", developed by Davies et al. "PROBOT" was designed for trans- urithral resection of the prostate and was clinically evaluated already in 1991 [14]. "PROBOT" followed a preoperative plan autonomously but under clinical supervision. The robot was initially based on a PUMA six-axis industrial robot equipped with two additional degrees of freedom, but subsequent iterations used customised mechatronic mechanisms that ensured the appropriate working envelope [59]. "PROBOT" is, again, a testament to the evolution of surgical systems from industrial manipulators to application-specific configurations. Moreover, as the first system of its generation, "PROBOT" demonstrated the complexity of soft-tissue surgery when undertaken without an intraoperative imaging modality or synergistic surgical manipulation. These two key characteristics are elements on which newer robotic systems are based.

One of the first endeavours to exploit these notions was "LARS" published by Taylor et al. in 1992. "LARS" was based on IBM's modified SCARA system, and was a telemanipulated robot for laparoscopic surgery. Its concept was to include several robotic arms for manipulating the surgical tools and the endoscope, transporting the surgeon from the operating table to a nearby operating console. The first evaluation of the system was as an endoscope manipulator [57].

The arrangement of several robotic arms to create a laparoscopic surgical robot was further evolved by Computer Motion Inc. and their "ZEUS" platform. "ZEUS" consisted of "AESOP" arms whose initial intended function was, again, to manoeuvre an endoscope. "ZEUS" was used surgically between 1998 and 2003, including the transatlantic tele-surgery "Operation Lindbergh" by Marescaux et al. [60]. The merger of Computer Motion Inc. and Intuitive Surgical Inc. led to "ZEUS" withdrawal from market in favour of the "da Vinci" platform, a teleoperated robot stemming from the research of Green et al. at the Stanford Research Institute [61]. The first application of "ZEUS" and the "da Vinci" was minimally invasive cardiac surgery [15], [62]. Since then, the "da Vinci" has found an extensive range of applications, with its primary use being radical prostatectomy [63].

Three characteristics of the "da Vinci" can be identified as main reasons for its surgical, as well as commercial success. First, the system utilises stereoendoscopes, and, hence, returns the lost depth perception to the surgeon. Second, the surgeon operates in an immersive environment, with his gaze directed towards his hands. This natural operating posture is preferred to looking at a video screen, since it restores hand-eye coordination. Perhaps its biggest innovation, however, is the seven-degree of freedom end-effectors (endowrist instruments) that mimic natural wrist/hand motions combined with motion scaling. These robotic wrists, inspired from the seminal 1998 publication of the "Black Falcon" from Madhani and Salisbury [73], restore the wrist articulation that is lost during laparoscopy. Hence, the "da Vinci" manages to restore the sense of sight with fully aligned visuomotor axes while providing increased surgical dexterity. Haptic feedback, even though identified as a crucial element to restore in laparoscopy as early as 1991 [74], is still a topic of ongoing research in both academic and industrial levels [75]. Newer laparoscopic surgery systems aim to improve the successful paradigm of "da Vinci" by focusing on reducing system volume and footprint 
TABLE I

EXAMPLES OF COMMERCIALISED SURGICAL RoBotS

\begin{tabular}{r|r|r|r} 
Robot Name & Procedure & Institution & Ref. \\
\hline \multicolumn{5}{|c}{ Stereotaxic Surgical Robots } \\
Neuromate & Neurosurgery & Renishaw Plc, FR & {$[41]$} \\
Pathfinder & Neurosurgery & Prosurgics, UK & {$[64]$} \\
SpineAssist & Neurosurgery & Mazor Robotics, USA & {$[46]$} \\
ROBODOC & Orthopaedics & Curexo Technology Corp., USA & {$[13]$} \\
AcroBot & Orthopaedics & MAKO Surgical Corp. USA & {$[53]$} \\
iBlock & Orthopaedics & Praxim Inc., FR & {$[56]$} \\
\multicolumn{5}{|c}{ Rigid Dextrous Robots for MIS } \\
\hline ZEUS & Heart Surgery & Computer Motion Inc, USA & {$[15]$} \\
da Vinci & Laparoscopy & Intuitive Surgical Inc, USA & {$[62]$} \\
Raven & Laparoscopy & Applied Dexterity Inc., USA & {$[65]$} \\
\multicolumn{5}{|c}{ Flexible Robots for MIS } \\
\hline CardioArm & Heart Surgery & Medrobotics Corp., USA & {$[66]$} \\
SPORT & Laparoscopy & Titan Medical Inc., CA & {$[67]$} \\
Anubis & Laparoscopy & Karl Storz GmbH, DE & {$[68]$} \\
Cobra & Laparoscopy & USGI Medical Inc., USA & {$[69]$} \\
EndoSAMURA & Laparoscopy & Olympus Medical Corp., JP & {$[6]$} \\
Magelan & Catheterisation & Hansen Medical Inc., USA & {$[70]$} \\
Amigo & Catheterisation & Catheter Robotics Corp., USA & {$[71]$} \\
Niobe & Catheterisation & Stereotaxis Inc., USA & {$[72]$} \\
CGCI & Catheterisation & Magnetecs Inc., USA & {$[72]$}
\end{tabular}

and on providing haptic feedback.

The "MiroSurge" system, developed by Hirzinger et al. of the German Aerospace Centre (DLR), Germany, uses a configuration similar to "ZEUS" and "LARS" [76]. Three to five robotic arms, named "MIRO", attach to the operating table, with their location specified based on the desired entry angle and location of the laparoscopic tools. Each arm weighs on the order of $10 \mathrm{~kg}$, making the system significantly light. Its wrist-equipped tools integrate a force/torque sensing to quantify tool/tissue interactions. A remote centre of motion around the fulcrum point is enforced programmatically rather than through the robot architecture, making the system more versatile.

"Raven" (Washington University, Seattle, United States), developed by Rosen and Hannaford et al., is also a lightweight surgical system with arms mounted directly on the operating table [77]. The developers of "Raven" have recently started providing the robot as an open platform for teaching and research, as well as for testing and development of new surgical mechanisms and software. Several international universities are already in possession of these systems [65]. A notable characteristic of "Raven" is that it employs a flexible endoscope for improved visibility [78]. Indeed, flexible surgical instruments and robots, as will be described in the following section, are rapidly gaining traction with increased effort being invested in the creation of platforms that can navigate around anatomical targets to reach deep-seated pathological locations.

Also of interest in MIS are smart surgical tools manually operated by the surgeon that augment his/hers surgical capabilities. "Steady Hand Robot", by Taylor et al. is one of the first robots of this category. Apart from enforcing safe and unsafe workspace regions via active constraints, it scales the motions and forces of the surgeon [34]. Another example, "Micron", developed by Riviere et al., minimises the surgeon's hand tremor and allows him/her to perform interventions with micrometre accuracy [79] (see Fig. 3(b)). Both "Steady Hand Robot" and "Micron" have ophthalmic surgery as their primary application. Other endeavours by Yang et al. focus on stabilising clinical motions for increased accuracy in endomicroscopy [80]. Hand-held robots are easily acceptable by surgeons since they resemble familiar tools and can be directly introduced in their workflow.

\section{Flexible Robots for MIS - $3^{\text {rd }}$ Generation}

MIS robots have improved surgical outcomes and reduced patient trauma, although the tangible economic impact and patient benefits still need to be established. For a technology that is still young and constantly evolving, perhaps one should be patient enough to wait until its relative maturity. Nevertheless, the current achievements set more demanding research-anddevelopment goals: further miniaturisation of the platform and reduction of trauma by minimising entry incisions to a single one, and further increase of dexterity to operate through natural pathways. These new interventional procedures are referred as NOTES (Natural Orifice Transluminal Endoscopic Surgery) and SILS (Single Port Laparoscopic Surgery).

Flexible robots are a technology promising to revolutionise both endoluminal and transluminal surgeries. Developed platforms comprise miniaturised mechatronic components to create arm-like assemblies with active shape control, and their flexibility allows insertion through natural orifices, e.g., transvaginally or transorally, and operation inside anatomical lumen, e.g., the stomach or the abdomen. Even though flexible robots are traced to the 1960s [81], the first flexible medical robot, an endoscope with the capability to control its shape along its entire length, did not appear until 1988. It was developed by Ikuta et al., and employed shape-memory alloys (SMA) pulling on a spring skeleton for bending in three directions [82]. Tendon-driven mechanisms were also explored early [83], with the primary focus always being endoscopy. These endeavours identified the biggest challenges in developing flexible surgical robots: (a) maximise their angular bending capability, (b) increase their ability to triangulate their arms for dextrous tissue manipulation, and (c) exercise significant 
forces on tissue. Several noteworthy flexible surgical robots are in development, and readers are referred to [28] for a review.

Flexible robots for laparoscopic interventions are most commonly based on connected articulated joints that form hyperredundant robots, or tendons that deform a skeleton by pulling. An example of the first approach is "CardioArm", which employs the three-degree-of-freedom joint developed by Choset et al. [66]. "CardioArm" is licensed by Medrobotics Corp. for minimally invasive heart and throat surgery. Another example, "i-Snake", developed by Yang et al., employs universal-type joints and has full retroflexion capability [38] as is necessary in certain interventions, e.g., tubal ligation in the pelvis or PEOM in the stomach with a NOTES approach. The robots provide a lumen for interchangable surgical tools.

Tendon-driven flexible robots can be made smaller. They do not possess discrete components but control their overall shape through traction on tendons running through an elastic backbone [84]. The tendons can be actuated by pulling, as, for example, in the tendon-driven bimanual flexible robot of Simaan et al. [67]. The initial application of this robot was throat surgery in a NOTES fashion [67], but with the technology being licensed by Titan Medical Inc., it is expected to find general usage in SILS. Other examples are the platforms "Anubis" by IRCAD and Karl Storz GmbH, Cobra by USGI Medical Inc., EndoSAMURAI by Olympus Medical Corp. [6], [68], [69], and the hybrid micro-motor tendon-driven flexible arms with articulated head in the bimanual platform of Yang et al. [7]. Other notable endeavours include the SPRINT platform, result of the EU consortium ARAKNES [85].

While it is possible in many surgical scenarios to obviate anatomical constraints, as, e.g., by insufflating the abdominal cavity, there are pathology locations where instruments need to be inserted along tortuous paths while manipulating tissue in confined anatomical regions including the heart, kidneys, or brain ventricles. The aforementioned flexible robots cannot be applied there due to their large diameter. The smallest flexible robots, with diameters on the order of a few millimetres, are steerable catheters and concentric tube robots.

Catheters, due to their flexibility, can navigate long paths of the human vasculature. Catheter-based interventions such as cardiac ablation for atrial fibrillation are becoming increasingly common [86]. Therein, a catheter is navigated through the femoral artery in the groin towards the heart where it performs cauterisation for arrhythmia treatment. Manually controlling the catheter is challenging and time consuming, and collaborative learning-based systems are being developed to increase operational accuracy [87]. Since fluoroscopy is used for visualisation, however, clinicians and patients are exposed to large amounts of radiation. Hence, steerable catheter systems that remove the clinician from the operating room are investigated, with mechanical and electromagnetic steering being the predominant solutions.

Mechanical steering is based on engineering flexible components to the catheter. SMAs can be used to create catheters with active shape control of their tip, as described by Patel et al. [88]. Thus, the catheter can be initially steered manually in a coarse fashion, and, subsequently, fine manipulations of the catheter tip can be applied. An alternative approach is to use tendons, similar to certain flexible robots, as is the operating principle of the commercial systems "Magellan" from Hansen Medical Inc. [70], and "Amigo" from Catheter Robotics Inc. [71]. Other mechanical steering systems operate by replicating the clinician's motions [89].

Electromagnetic steering is based on equipping the catheter with magnetic parts and applying electromagnetic forces and torques for its navigation. Such concepts were proposed in the early stages of the field [2], [3], but the vast technological challenges have only recently made them realisable. "Niobe" from Stereotaxis Inc. performs steering by moving large permanent magnets, and is used for numerous atrial fibrillation procedures worldwide [72]. The system requires permanent magnetic shielding and safety measures similar to MRI suites. Alternative systems employ stationary electromagnets and shape the electromagnetic field through current control, thus requiring less rigorous safety mechanisms. The "CGCI" system from Magnetecs Inc., which has obtained CE mark and is currently deployed in several centres worldwide, employs an array of eight electromagnets [90] and allows full 6 DOF control of a catheter tip. Finally, Martel et al. propose using the gradient field of MRI scanners to deflect and navigate catheters [91]. The integrated imaging capabilities of the MRI are a significant benefit, but the limited control over the generated magnetic fields do not allow control over the torque applied on the catheter.

Even though robotic steering does limit the clinician's exposure to fluoroscopy-based radiation by removing him/her from the operating room [92], using MRI completely eliminates irradiation of both clinician and patient. Hence, similar to the evolution of stereotaxic robots (Sec. II-B), there is extensive effort to make catheter guidance systems MRI-compatible [93]. Force feedback, considered important also in catheterbased interventions [94], can be provided using mechanisms based on fibre optics [95], [96]. There is ongoing effort to make these force sensors MRI-compatible [95]. Increased accuracy, intraoperative visualisation, and haptic feedback will allow ultra-minimally invasive interventions without current catheterisation challenges. The main drawback of catheters, however, i.e., their inability to apply large forces, prohibits their use in complex operations, e.g., in leaflet repair. Concentric tube robots are stiff but compliant continuum robots with catheter-size dimensions that are suited for such interventions.

Concentric tube robots comprise precurved concentric superelastic tubes that rotate and translate with respect to each other. The interaction of the different curvatures gives rise to the final robot shape, which can be controlled to allow navigation of tortuous paths. Concentric tube robots were simultaneously and independently introduced in 2006 by Dupont et al. [97] and Webster et al. [98]. Even though proposed for several surgical interventions such as neurosurgery [99] and cardiac surgery [100], only in 2013 have they been applied in vivo, in porcine heart surgery [100]. The procedure was observed using ultrasound and fluoroscopy. The robot was inserted through a neck incision and was extended through the jugular vein and the superior vena cava towards the left atrium, to perform patent foramen ovale closure. The lack of high-quality interoperative visualisation and haptic feedback 
complicated surgery, but research on force sensing based on Fiber-Bragg Gratings [101], [102] and MRI-compatibility [103] may provide touch and sight to these robots as well.

Flexible robots can reach certain pathology locations ultraminimally invasively. Recent advances provide increased dexterity and bring harmless intraoperative imaging and force feedback even to the smallest of continuum robots. Given the rise of manually steered flexible laparoscopic tools, it can be anticipated that in the coming decades continuum robots will become valuable tools of the surgical workflow. Due to manufacturing limitations inherent to their structure, however, flexible robots cannot be made arbitrarily long and are thus limited with regards to their insertion depth. Steerable capsule endoscopes and microrobots promise untethetered intracorporeal navigation and the highest interventional precision.

\section{E. Untethered Microsurgeons - $4^{\text {th }}$ Generation}

The vision of tiny devices roaming inside the human body to detect and fight pathologies has been inspirational for science fiction movies and science talks. In one of the most prominent seminars, "There's plenty of room at the bottom" by Richard Feynman (Dec. 1959) [106], the vision was crystallised in "[...] swallow[ing] the surgeon; [putting] the mechanical surgeon inside the blood vessel, [so that] it goes to the heart [to] look around". Untethered microsurgeons that navigate intracorporeally by penetrating tissue or using anatomical pathways realise this vision and constitute the smallest medical robots.

Capsule endoscopes are millimetre-scale devices that, at their current commercial state, explore the gastrointestinal (GI) tract. They perform diagnosis through image acquisition and are passively propelled based on peristalsis [107]. Their passive locomotion prohibits them from reexamining lesions or intervening [26]. Steerable capsule endoscopes, on the other hand, can navigate using onboard locomotion or external energy transfer mechanisms [26]. Biomimetic locomotion principles like insect-like legged or inchworm locomotion have been proposed by several research groups, e.g., by Sitti et al. [108], Dario et al. [109], and Park et al. [110], with several technologies having been tested ex vivo and in vivo. Similar to catheter steering, effort is invested in magnetic steering, either with dedicated electromagnetic systems as in [111] or in the Olympus Medical Corp./Siemens AG. partnership [112], MRI scanners [113], or mobile permanent magnets [114][116]. Capsule endoscopes coupled with thermally actuated nanogrippers can perform localised biopsies [117] and wireless colon insuflation [118], or deliver drugs [37]. The capsules can be localised within the body by exploiting the magnetic field they produce [119], [120] or by ultrasound imaging [121], and can, thus, be servoed to the desired anatomical sites. Recent technological advances by Ishiyama et al. and Abbott et al. demonstrate that screw-like capsules can burrow in tissue, thus expanding their operational capabilities [122], [123]. Ongoing research will result in versatile capsule platforms for disease treatment and intervention.

To achieve navigation in the smallest sections of human anatomy, i.e., the spinal channel or capillaries, miniaturisation must be pushed even further, reaching the domain of microsurgeons. Microrobotics is one of the newest research areas in surgical robotics. Microrobots have been proposed for various applications, e.g., arterial plaque removal [10], intravascular drug delivery [124], intraocular drug delivery [125], drug delivery in the brain [126]. A thorough review of state-of-the-art systems can be found in [25]. Due to this domain's youth, few systems have been evaluated in vivo; notable examples include intraocular microrobot mobility tests in rabbits by Nelson et al. [105] (see Fig. 3(c)), and guidance in the carotid artery of swine, and rabbits, by Martel et al. [127], and Park et al. [128], respectively.

At small scales, untethered power transfer is extremely challenging since batteries cannot be sufficiently miniaturised. Hence, external powering mechanisms are investigated. Even though electrostatic fields [129], attached flagellated bacteria [130]-[132], and thermal gradients [133] have been proposed for microrobot propulsion, the most common actuation method is based on arrays of electromagnets and electromagnetic field control due to the favourable scaling of electromagnetic forces and torques with respect to device volume [134].

Visualisation presents an additional challenge in microrobotics. The most commonly used imaging modality, endoscopy, is inapplicable since it involves incisions that are orders of magnitude larger that the microdevices themselves. Fluoroscopy has been occasionally employed [128], but since the ionising radiation poses risks to patients and clinicians alternative imaging methods need to be employed, with the greatest challenges arising from the facts that: 1) increased resolution is required to detect micrometre-scale devices, and 2) the imaging systems should integrate with bulky external powering mechanisms, preventing, for example, the use of PET/SPECT scanners. A promising approach involves using MRI scanners, since they enable both magnetic actuation and visualisation; the magnetic microrobots appear in the images as detectable artefacts [9]. Alternatively, when the operating area is externally observable, as in ophthalmology, steering systems can be coupled with optical microscopes [105], [135].

In summary, certain challenges relating to manufacturing and functionalisation of tiny devices have been addressed and benchtop experiments have been performed. Successful coupling of real-time imaging modalities, high volume powering, and robust controllers with microdevices will make the latter indispensable tools of future operating rooms, capable of treating pathologies with ultra-high precision at early stages of the disease development.

\section{Surgical Robotics: Future}

Over the past 60 years, the field of surgical robots has evolved into a dynamic area of research and development, with versatile and robust mechanisms already being deployed in several operating theatres worldwide. The previous sections allow us to identify several trends and directions of the field.

It is now acknowledged that robots should be lightweight, compliant, and allow for close interaction between clinicians, patients, and the robot itself [76]. Surgical robots, once off-theshelf industrial manipulators, are becoming smaller following the advances in manufacturing and capitalising on the success 

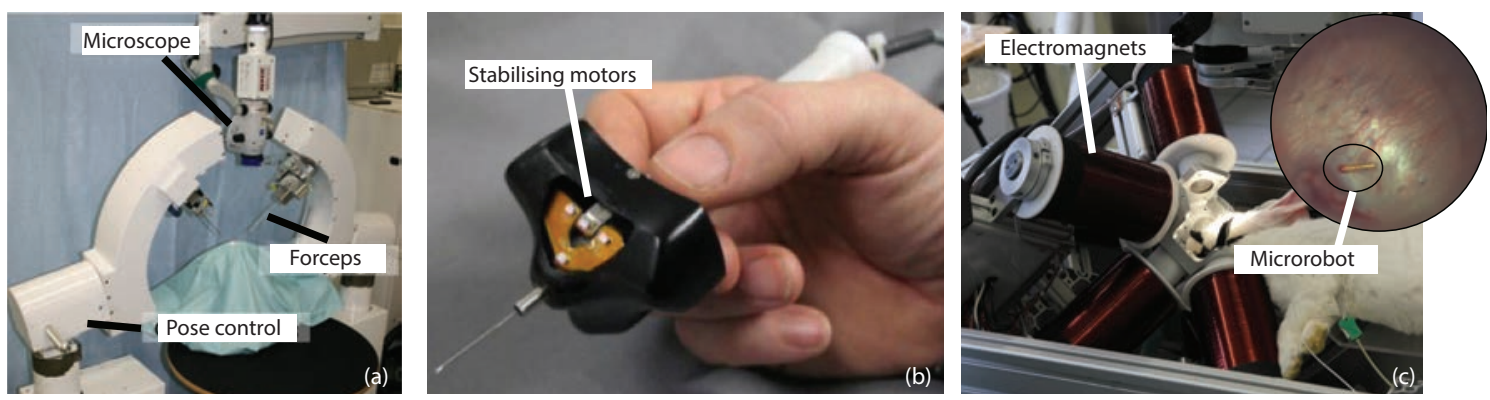

Fig. 3. Retinal surgery requires impressive dexterity and micrometre accuracy. Surgical platforms range from (a) large-scale remote-centre-of-motion teleoperated systems that replicate and scale clinician's motion: image adapted from Mitsuishi et al., [104], to (b) hand-held stabilising tools that increase surgical performance by measuring and filtering hand tremor: image adapted from Riviere et al., [79], and (c) wireless electromagnetically navigated microdevices for drug delivery: image adapted from Nelson et al., [105].

of MEMS devices [22]. Future surgical robots will be mounted on the operating table directly and will be reconfigurable with ease by non-technical personnel. Even smaller robots will be rigidly attached to the patient, or will be implanted in the human body to detect pathologies and intervene intracorporealy. Fig. 3 illustrates the miniaturisation in ophthalmic surgery.

Robots are now able to synergistically operate with surgeons, steering away from simple execution of preprogrammed plans. Not only increased surgical accuracy and superhuman dexterity is achieved, but high quality intraoperative imaging and force feedback as well. As a result, the senses of sight and touch that were deprived from surgeons with the advent of laparoscopic techniques are now returning in the operating room. MRI-compatible surgical robots will allow intraoperative 3D visualisation of anatomical volumes, providing clinicians with unprecedented levels of anatomical details. Hardware and software constraints will guarantee safe instrument manipulation both with regards to entry incisions and safe/unsafe anatomical regions. Implementation of force sensors along the tool and end-effectors of robots will be an additional pillar for safety and increased surgical success. Decades of research have established the limitations of both surgeons and robots, and, we can now develop robotic systems that complement rather than alter the surgical workflow. This leads to more acceptable systems with increased penetration potential.

Acceptability, however, is one aspect of the accessibility issues that surgical robots face. Despite their acknowledged advantages, their high cost prohibits wide adoption, especially in emerging economies worldwide. Effort is already invested in designing lightweight systems, thus limiting material costs. We expect that $3 \mathrm{D}$ printing technologies will further minimise surgical robot manufacturing costs and increase their accessibility. There already exist several 3D printed medical devices, albeit mainly for rehabilitation purposes [136], [137], and this trend is expected to propagate in the surgical robotics field. More important, perhaps, are the capital and resources associated with acquiring certification. The long and stringent process is a significant challenge for research groups planning to take a conceptual robotic design all the way to clinical translation. Given the current market space in medical robotics, new synergies need to be created such that a balanced ecosystem is created, which promotes innovation whilst focusing on tangible patient benefit and realistic social-economic impact

Our apprehension of surgical robotics may shift in the coming years as well. With increased research in wireless power transfer, mainly driven through advances in electromagnetic steering and control, surgical robots may be viewed as systems possessing wireless links between their actuation components, e.g., the electromagnets, and their end-effector, e.g., the steerable catheter, microdevice, or articulated joints for intraabdominal surgery [138].

Future surgical robots will interact with pathology sites at microscopic levels to deliver highly concentrated drugs in a localised fashion, thus reducing side-effects and increasing treatment success. Up to now, successful research in surgical robots required the combined efforts and skill sets of engineers and clinicians. As surgical devices become smaller and approach the nanometre domains, thorough understanding of the variety of forces that operate at these scales will be necessary. There are several fundamental differences pertaining to the interaction of tiny devices with tissue, and electrostatic forces, van der Waals forces, and chemical interactions become significant. Their modelling will require incorporating contributions from the fields of physics and chemistry. Moreover, to create devices that interact with patients at a microscopic scale research teams may need to expand and include molecular biologists. Indeed, if microsurgical robots are to detect and combat pathologies, e.g., cancer [131], or if they are to deliver engineered stem cells [139] or bind to arterial plaque [140], concrete knowledge of biological processes is necessary. Finally, chemical and biological reactions themselves may allow robots to harness power from their immediate environment. Given the challenges relating to force application at the microscale discussed in Sec. II-E, further research is required before reaching a universally acceptable solution.

Grand challenges in surgical robotics are also identified in [141]. It is suggested that larger research centres that can pool their resources may be formed to make acquisition of expensive high-end infrastructure possible. Indeed, in light of recent research budget sequestrations in the USA [142] and Europe [143] and their hindering effects on research output [144], inter departmental and cross-institutional collaboration is critical. As the commercialised platforms of Table I demonstrate, however, the potential clinical and commercial return of 
surgical robotics can be substantial. Thus, together with their undeniable societal impact, surgical robots can have a great future in years to come.

\section{COnClusions}

The advent of laparoscopy made routine open-surgery interventions complex, and already complex operations impossible. We are now at a state where surgical robotics have succeeded in facilitating MIS by granting even the average surgeon superhuman capabilities. We now possess the technology not only to increase success rates for existing interventions, but to also carry out conceptually new interventions. It has been understood that only through human/robot synergies, wherein decision making lies in the hands of the surgeon and manipulation in the hands of the robot, the highest patient benefit can be achieved. Systems are becoming smaller, and less expensive. In the years to come, an ever increasing arsenal of intracorporeal devices will appear and will make possible the fight of pathologies at a molecular level.

In his talk, Feynman stated that the design of the swallowable surgeon was a task that he "would leave [...] up to [us]". The inspiring progress of the surgical robotics field in the last 60 years demonstrates that our community is well capable of designing and developing the robotic surgical systems that were previously only present in the imagination of the pioneers.

\section{REFERENCES}

[1] J. Driller and G. Neumann, "An electromagnetic biospy device," IEEE Trans. Biomedical Engineering, vol. 14, no. 1, pp. 52-53, 1967.

[2] J. Molcho, H. Z. Karny, E. H. Frei, and M. Harden, "Selective cerebral catheterization," IEEE Trans. Biomedical Engineering, vol. 17, no. 2, pp. 134-140, 1970.

[3] D. B. Montgomery, R. J. Weggel, M. J. Leupold, S. B. Yodh, and R. L. Wright, "Superconducting magnet system for intravascular navigation," J. Applied Physics, vol. 40, no. 5, pp. 2129-2132, 1969.

[4] K. G. Engelhardt, "An overview of health and human service robotics," Robotics and Autonomous Systems, vol. 5, pp. 205-226, 1989.

[5] _ , "Current status and future prospect for robotic technology in health care delivery," Central Nervous System Trauma, vol. 3, no. 1, pp. 111-126, 1986.

[6] S. N. Shaikh and S. Thompson, "Natural orifice translumenal surgery: flexible platform review," World J. Gastrointestinal Surgery, vol. 2, no. 6, pp. 210-216, 2010.

[7] J. Shang, C. J. Payne, J. Clark, D. P. Noonan, K.-W. Kwok, A. Darzi, and G.-Z. Yang, "Design of a multitasking robotic platform with flexible arms and articulated head for minimally invasive surgery," IEEE Int. Conf. Robotics and Automation, pp. 1988-1993, 2012.

[8] K. Ishiyama, M. Sendoh, A. Yamazaki, and K. I. Arai, "Swimming micro-machine driven by magnetic torque," Sensor and Actuators A: Physical, vol. 91, no. 1-2, pp. 141-144, 2001.

[9] O. Felfoul, J.-B. Mathieu, G. Beaudoin, and S. Martel, "In vivo MRtracking based on magnetic signature selective excitation," IEEE Trans. Medical Imaging, vol. 27, no. 1, pp. 28-35, 2008.

[10] C. Yu, J. Kim, H. Choi, J. Choi, S. Jeong, K. Cha, J. Park, and S. Park, "Novel electromagnetic actuation system for three-dimensional locomoation and drilling of intravascular microrobot," Sensor and Actuators A: Physical, vol. 161, pp. 297-304, 2012.

[11] Y. S. Kwoh, J. Hou, E. A. Jonckheere, and S. Hayati, "A robot with improved absolute positioning accuracy for CT guided stereotactic brain surgery," IEEE Trans. Biomedical Engineering, vol. 35, no. 2, pp. $153-160,1988$.

[12] J. Pransky, "ROBODOC - surgical robot success story," Industrial Robot, vol. 24, no. 3, pp. 231-233, 1997

[13] H. A. Paul, W. L. Bargar, B. Mittelstadt, B. Musits, R. H. Taylor, P. Kazanzides, Z. Zuhars, B. Williamson, and W. Hanson, "Development of a surgical robot for cementless total hip arthroplasty," Clinical Orthopaedics, vol. 285, pp. 57-66, 1992.
[14] W. S. Ng, B. L. Davies, R. D. Hibberd, and A. G. Timoney, "Robotic surgery: a first-hand experience in transurethral resection of the prostate," IEEE Engineering in Medicine and Biology Magazine, vol. 12 , no. 1 , pp. 120-125, 1993.

[15] A. Carpentier, D. Loulmet, B. Aupecle, A. Berrebi, and J. Rellard, "Computer-assisted cardiac surgery," Lancet, vol. 353, pp. 379-380, 1999.

[16] J. M. Dolan, M. B. Friedman, M. K. Nagurka, and J. K. Gotow, "A robot in an operating room: a bull in a china shop?" IEEE Int. Conf. Engineering in Medicine and Biology, pp. 1096-1097, 1987.

[17] R. H. Taylor, H. A. Paul, P. Kazanzides, B. Mittelstadt, W. Hanson, Z. Zuhars, B. Williamson, B. Musits, E. Glassman, and W. L. Bargar, "Taming the bull: safety in a precise surgical robot," IEEE Int. Conf. Advanced Robotics, vol. 1, pp. 865-870, 1991.

[18] R. D. Howe and Y. Matsuoka, "Robotics for surgery," Annual Review of Biomedical Engineering, vol. 01, pp. 211-240, 1999.

[19] B. L. Davies, "A review of robotics in surgery," J. Engineering in Medicine, vol. 214, pp. 129-140, 2000.

[20] R. H. Taylor and D. Stoianovici, "Medical robotics in computerintegrated surgery," IEEE Trans. Robotics and Automation, vol. 19, no. 5, pp. 765-781, 2003.

[21] D. B. Camarillo, T. M. Krummer, and J. K. Salisbury, "Robotic technology in surgery: past, present, and future," American J. Surgery, vol. 188, pp. 2S-15S, 2004.

[22] K. J. Rebello, "Applications of MEMS in surgery," Proc. IEEE, vol. 92, no. 1, pp. 43-55, 2004.

[23] A. Menciassi, M. Quirini, and P. Dario, "Microrobotics for future gastrointenstinal endoscopy," Minimally Invasive Therapy, vol. 16, no. 2, pp. 91-100, 2007.

[24] G. Dogangil, B. L. Davies, and F. Rodriguez y Baena, "A review of medical robotics for minimally invasive soft tissue surgery," $J$. Engineering in Medicine, vol. 224, no. 5, pp. 653-679, 2010.

[25] B. J. Nelson, I. K. Kaliakatsos, and J. J. Abbott, "Microrobots for minimally invasive medicine," Annual Review of Biomedical Engineering, vol. 12 , pp. $55-85,2010$.

[26] G. Ciuti, A. Menciassi, and P. Dario, "Capsule endoscopy: from current achievements to open challenges," IEEE Reviews in Biomedical Engineering, vol. 4, pp. 59-72, 2011.

[27] R. A. Beasley, "Medical robots: current systems and research directions," J. Robotics, 2012.

[28] V. Vitiello, S.-L. Lee, T. P. Cundy, and G.-Z. Yang, "Emerging robotic platforms for minimally invasive surgery," IEEE Reviews in Biomedical Engineering, vol. 6, pp. 111-126, 2013.

[29] R. Walker Jr., "The first laparoscopic cholecystectomy," J. Society of Laparoscopic Sugerons, vol. 5, no. 1, pp. 89-94, 2001.

[30] F. Keus, J. A. de Jong, H. G. Gooszen, and C. J. van Laarhoven, "Laparoscopic versus open cholecistectomy for patients with symptomatic cholecystolithiasis," Cochrane Library, vol. 1, pp. 1-156, 2009.

[31] Y. S. Tanagho, G. L. Andriole, A. G. Paradis, K. M. Madison, S. S. Gurdarshan, J. E. Varela, and B. M. Benway, "2D versus 3D visualization: impact on laparoscopic proficiency using the fundamentals of laparoscopic surgery skill set," J. Laparoendoscopic and Advanced Surgical Techniques, vol. 22, no. 9, pp. 865-870, 2012.

[32] A. G. Gallagher, N. McClure, J. McGulgan, K. Ritchie, and N. P. Sheehy, "An ergonomic analysis of the fulcrum effect in the acquisition of endoscopic skills," Endoscopy, vol. 30, no. 7, pp. 617-620, 1998.

[33] A. D. Jagtap and C. N. Riviere, "Applied force during vitreoretinal microsurgery with handheld instruments," IEEE Int. Conf. Engineering in Medicine and Biology, pp. 2771-2773, 2004.

[34] R. H. Taylor, P. S. Jensen, L. Whitcomb, A. Barnes, R. Kumar, D. Stoianovici, P. Gupta, Z. X. Wang, E. de Juan, and L. Kavoussi, "A steady-hand robotic system for microsurgical augmentation," Int. J. Robotics Research, vol. 18, no. 12, pp. 1201-1210, 1999.

[35] M. Ho, A. B. McMillan, J. M. Simard, R. Gullapalli, and J. P. Desai, "Toward a meso-scale SMA-actuated MRI-compatible neurosurgical robot," IEEE Trans. Robotics, vol. 28, no. 1, pp. 213-222, 2012.

[36] S. Martel and M. Mohammadi, "A robotic micro-assembly process inspired by the construction of the ancient pyramids and relying on several thousand flagellated bacteria acting as micro-workers," IEEE/RSJ Int. Conf. Intelligent Robots and Systems, pp. 426-427, 2009.

[37] S. Yim and M. Sitti, "Design and rolling locomotion of a magnetically actuated soft capsule endoscope," IEEE Trans. Robotics, vol. 28, no. 1, pp. 183-194, 2012.

[38] R. C. Newton, D. P. Noonan, V. Vitiello, J. Clark, C. J. Payne, J. S. Shang, M. Sodergren, A. Darzi, and G.-Z. Yang, "Robot-assisted transvaginal peritoneoscopy using confocal endomicroscopy: a feasi- 
bility study in a porcine model," Surgical Endoscopy, vol. 26, no. 9, pp. 2532-2540, 2012.

[39] N. A. Patronik, T. Ota, M. A. Zenati, and C. N. Riviere, "A miniature mobile robot for navigation and positioning of the beating heart," IEEE Trans. Robotics, vol. 25, no. 5, pp. 1109-1124, 2009.

[40] United Press International, "Robot used in brain surgery," Chicago Tribune, April 19, 1985.

[41] A. L. Benabid, P. Cinquin, S. Lavalle, J. F. Le Bas, J. Demongeot, and J. de Rougemont, "Computer-driven robot for stereotactic surgery connected to ct scan and magnetic resonance imaging," Applied Neurophysiology, vol. 50, pp. 153-154, 1987.

[42] A. Kato, T. Yoshimine, T. Hayakawa, Y. Tomita, T. Ikeda, M. Mitomo, K. Harada, and H. Mogami, "A frameless, armless navigational system for computer-assisted neurosurgery: technical note," J. Neurosurgery, vol. 74 , no. 5 , pp. 845-849, 1991.

[43] D. Glauser, H. Fankhauser, M. Epitaux, J.-L. Hefti, and A. Jaccottet, "Neurosurgical robot Minerva: first results and current developments," Computer Aided Surgery, vol. 1, no. 5, pp. 266-272, 1995.

[44] G. R. Sutherland, P. B. McBeth, and D. F. Louw, "NeuroArm: an MR compatible robot for microsurgery," Int. Congress Series, vol. 1256, pp. 504-508, 2003.

[45] L. Joskowicz, R. Shamir, M. Freiman, E. Zehavi, F. Umansky, and Y. Shoshan, "Image-guided system with miniature robot for precise positioning and targeting in keyhole neurosurgery," Computer Aided Surgery, vol. 11, no. 4, pp. 181-193, 2006.

[46] D. P. Devito, L. Kaplan, R. Dietl, M. Pfeiffer, D. Horne, S. Silberstein, M. Hardenbrook, G. Kiriyanthan, Y. Barzilay, and A. Bruskin, "Clinical acceptance and accuracy assessment of spinal implants guided with spineassist surgical robot: a retrospective study," Spine, vol. 35, no. 24, pp. 2109-2115, 2010.

[47] D. Stoianovici, K. Cleary, A. Patriciu, D. Mazilu, A. Stanimir, N. Craciunoiu, V. Watson, and L. Kavoussi, "AcuBot: a robot for radiological intervention," IEEE Trans. Robotics, vol. 19, no. 5, pp. 927-930, 2003.

[48] K. Chinzei, N. Hata, F. A. Jolesz, and R. Kikinis, "MR compatible surgical assist robot: system integration and preliminary feasibility study," Int. Conf. Medical Image Computing and Computer Assisted Intervention, pp. 921-930, 2000.

[49] A. Melzer, B. Gutmann, T. Remmele, R. Wolf, A. Lukoscheck, M. Bock, H. Bardenheuer, and H. FIscher, "INNOMOTION for percutaneous image-guided interventions," IEEE Engineering in Medicine and Biology Magazine, pp. 66-73, May/June 2008.

[50] A. Krieger, R. C. Susil, C. Ménard, J. A. Coleman, G. Fichtinger, E. Atalar, and L. L. Whitcomb, "Design of a novel MRI compatible manipulator for image guided prostate interventions," IEEE Trans. Biomedical Engineering, vol. 52, no. 2, pp. 306-313, 2005.

[51] J. Tokuda, S. S.-E. Song, G. Fischer, I. Iordachita, R. Seifabadi, N. B. Cho, G. Fichtinger, C. M. Tempany, and N. Hata, "Preclinical evaluation of mri-compatible pneumatic robot for angulated needle placement in prostate interventions," Computer-assisted Radiology and Surgery, 2012

[52] P. Vartholomeos, C. Bergeles, L. Qin, and P. E. Dupont, "An MRIpowered and controlled actuator technology for tetherless robotic interventions," Int. J. Robotics Research, 2013, accepted.

[53] B. L. Davies, S. J. Harris, W. J. Lin, R. D. Hibberd, R. Middleton, and J. C. Cobb, "Active compliance in robotic surgery - the use of force control as a dynamic constraint," J. Engineering in Medicine, vol. 211, pp. 285-291, 1997.

[54] A. Bettini, P. Marayong, S. Lang, A. M. Okamura, and G. D. Hager, "Vision-assisted control for manipulation using virtual fixtures," IEEE Trans. Robotics, vol. 20, no. 6, pp. 953-966, 2004.

[55] B. C. Becker, R. A. MacLachlan, L. A. Lobes, G. D. Hager, and C. N. Riviere, "Vision-based control of a handheld surgical micromanipulator with virtual fixtures," IEEE Trans. Robotics, vol. 29, no. 3, pp. 674683, 2013.

[56] C. Plaskos, P. Cinquin, S. Lavalle, and A. J. Hodgson, "Praxiteles: a miniature bone-mounted for minimal access total knee arthroplasty," Int. J. Medical Robotics and Computer Assisted Surgery, vol. 1, no. 4, pp. $67-79,2005$

[57] R. H. Taylor, J. Funda, B. Eldridge, S. Gomory, K. Gruben, D. LaRose, M. Talamini, L. Kavoussi, and J. Anderson, "A telerobotic assistant for laparoscopic surgery," IEEE Engineering in Medicine and Biology Magazine, vol. 14, no. 3, pp. 279-288, 1995.

[58] P. Finlay and M. H. Ornstein, "Controlling the movement of a surgical laparoscope," IEEE Engineering in Medicine and Biology Magazine, vol. 14, no. 3, pp. 289-291, 1995.

[59] S. J. Harris, F. Arambula-Cosio, R. D. Mei, Q. Hibberd, B. L. Davies, J. E. A. Wickham, N. M. S., and B. Kundu, "The Probot - an active robot for prostate resection," J. Engineering in Medicine, vol. 211, no. H4, pp. 317-326, 1997.

[60] J. Marescaux, J. Leroy, M. Gagner, F. Rubino, D. Mutter, M. Vix, S. E. Butner, and M. K. Smith, "Transatlantic robot-assisted telesurgery," Nature, vol. 413, no. 6854, pp. 379-380, 2001.

[61] P. S. Green, J. W. Hill, J. F. Jensen, and A. Shah, "Telepresence surgery," IEEE Engineering in Medicine and Biology Magazine, vol. 14 , no. 3, pp. 324-329, 1995.

[62] G. S. Guthart and J. K. Salisbury, "The Intuitive telesurgery system: overview and application," IEEE Int. Conf. Robotics and Automation, pp. $618-621,2000$.

[63] V. Ficarra, S. Cavalleri, G. Novara, M. Aragona, and W. Artibani, "Evidence from robot-assisted laparoscopic radical prostatectomy: a systematic review," European Urology, vol. 51, pp. 45-46, 2007.

[64] P. S. Morgan, T. Carter, S. Davis, A. Sepehri, J. Punt, P. Byrne, A. Moody, and P. Finlay, "The application accuracy of the pathfinder neurosurgical robot," Int. Congress Series, vol. 1256, pp. 561-567, 2003.

[65] B. Hannaford, J. Rosen, M. B. Friedman, H. King, P. Roan, C. Lei, D. Glozman, M. Ji, S. Nia Kosari, and W. Lee, "Raven-ii: an open platform for surgical robotics research," IEEE Trans. Biomedical Engineering, vol. 60, no. 4, pp. 954-959, 2013.

[66] E. Shammas, A. Wolf, and H. Choset, "Three degrees-of-freedom joint for spatial hyper-redundant robots," Mechanism and Machine Theory, no. 41, pp. 170-190, 2006.

[67] N. Simaan, K. Xu, W. Wei, A. Kapoor, P. Kazanzides, R. H. Taylor, and P. Flint, "Design and integration of a telerobotic system for minimally invasive surgery of the throat," Int. J. Robotics Research, vol. 28, no. 9, pp. 1134-1153, 2009.

[68] Karl Storz GmbH. (2013) Anubis. [Online]. Available: https://www.karlstorz.com/cps/rde/xchg/SID-855363E7-43052894/ karlstorz-en/hs.xsl/8872.htm

[69] S. J. Bardaro and L. Swanstrom, "Development of advanced endoscopes for Natural Orifice Transluminal Endoscopic Surgery (NOTES)," Minimally Invasive Therapy, vol. 15, no. 6, pp. 378-383, 2006.

[70] J. Bismuth, E. Kashef, N. Cheshire, and A. B. Lumsden, "Feasibility and safety of remote endovascular catheter navigation in a porcine model," J. Endovascular Therapy, vol. 18, no. 2, pp. 243-249, 2011.

[71] E. M. Khan, W. Frumkin, G. A. Ng, S. Neelagaru, F. M. AbiSamra, J. Lee, M. Giudici, D. Gohn, R. A. Winkle, J. Sussman, B. P. Knight, A. Berman, and H. Calkins, "First experience with a novel robotic remote catheter system: Amigo mapping trial," J. Interventional Cardiology and Electrophysiology, vol. 37, pp. 12-129, 2013.

[72] S. Ernst, F. Ouyang, C. Linder, K. Hertting, F. Stahl, J. Chun, H. Hachiya, D. Bänsch, M. Antz, and K.-H. Kuck, "Initial experience with remote catheter ablation using a novel magnetic navigation system," Circulation, vol. 109, no. 12, pp. 1472-1475, 2004.

[73] A. J. Madhani, G. Niemeyer, and J. K. Salisbury, "The black falcon a teleoperated surgical instrument for minimally invasive surgery," IEEE/RSJ Int. Conf. Intelligent Robots and Systems, pp. 936-944, 1998.

[74] B. Preising, T. C. Hsia, and B. Mittelstadt, "A literature review: robotics in medicine," IEEE Engineering in Medicine and Biology Magazine, vol. 10, no. 2, pp. 13-22, 1991.

[75] S. J. Blumenkranz and D. Q. Larkin, "Force sensing for surgical instruments," US Patten App. 11/864, 974, 2007.

[76] U. Hagn, R. Konietschke, A. Tobergte, M. Nickl, S. Jörg, B. Kübler, G. Passig, M. Gröger, F. Fröhclich, U. Seibold, L. Le-Tien, A. AlbuSchäffer, A. Nothhelfer, F. Hacker, M. Grebenstein, and G. Hirzinger, "DLR MiroSurge: a versatile system for research in endoscopic telesurgery," Int. J. Computer Assisted Radiology and Surgery, vol. 5, no. 2, pp. 183-193, 2010 .

[77] M. J. H. Lum, D. C. W. Friedman, G. Sankaranarayanan, H. King, K. Fodero, R. Leuschke, B. Hannaford, J. Rosen, and M. N. Sinanan, "The raven: design and validation of a telesurgery system," Int. J. Robotics Research, vol. 28, no. 9, pp. 1183-1197, 2009.

[78] C. A. Velasquez, H. King, B. Hannaford, and W. J. Yoon, "Development of a flexible imaging probe integrated to a surgical telerobot system: preliminary remote control test and probe design," IEEE Int. Conf. Biomedical Robotics and Biomechatronics, pp. 894-898, 2012.

[79] R. A. MacLachlan, B. C. Becker, J. C. Tabarés, G. W. Podnar, L. A. Lobes, and C. N. Riviere, "Micron: an actively stabilized handheld tool for microsurgery," IEEE Trans. Robotics, vol. 28, no. 1, pp. 195-212, 2012.

[80] W. T. Latt, R. C. Newton, M. Visentini-Scarzanella, C. J. Payne, D. P. Noonan, J. S. Shang, and G.-Z. Yang, "A hand-held instrument to maintain steady tissue contact during probe-based confocal laser 
endomicroscopy," IEEE Trans. Biomedical Engineering, vol. 58, no. 9, pp. 2694-2703, 2011.

[81] V. Anderson and R. Horn, "Tensor arm manipulator design," AMSE Transactions, vol. 67-DE-57, no. 2, pp. 1-12, 1967.

[82] K. Ikuta, M. Tsukamoto, and H. Shigeo, "Shape memory alloy servo actuator system with electric resistance feedback and application for active endoscope," IEEE Int. Conf. Robotics and Automation, pp. 427430, 1988

[83] R. H. Sturges and S. Laowattana, "A flexible, tendon-controlled device for endoscopy," Int. J. Robotics Research, vol. 12, no. 2, pp. 121-131, 1993.

[84] D. B. Camarillo, C. F. Milne, C. R. Carlson, M. R. Zinn, and J. K. Salisbury, "Mechanics modeling of tendon-driven continuum manipulators," IEEE Trans. Robotics, vol. 24, no. 6, pp. 1262-1273, 2008.

[85] M. Piccigallo, U. Scarfogliero, C. Quaglia, G. Petroni, P. Valdastri, A. Menciassi, and P. Dario, "Design of a novel bimanual robotic system for single-port laparoscopy," IEEE/ASME Trans. Mechatronics, vol. 15, no. 6, pp. 871-878, 2010.

[86] R. Cappato, H. Calkins, S.-A. Chen, W. Davies, Y. Iesaka, J. Kalman, Y.-H. Kim, G. Klein, A. Natale, D. Packer, A. Skanes, A. Ambrogi, and E. Biganzoli, "Updated worldwide survery on the methods, efficay, and safety of catheter ablation for human atrial fibrillation," Circulation: Arrhtymia and Electrophyisiology, vol. 3, no. 1, pp. 32-38, 2010.

[87] H. Rafii-Tari, J. Liu, S.-L. Lee, C. Bicknell, and G.-Z. Yang, "Learningbased modeling of endovascular navigation for collaborative robotic catheterization," Int. Conf. Medical Image Computing and Computer Assisted Intervention, pp. 369-377, 2013.

[88] J. Jayender and R. V. Patel, "Robot-assisted active catheter insertion: algorithms and experiments," Int. J. Robotics Research, vol. 28, no. 9, pp. 1101-1117, 2009.

[89] Y. Thakur, D. W. Holdsworth, and M. Drangova, "Characterization of catheter dynamics during percutaneous transluminal catheter procedures," IEEE Trans. Biomedical Engineering, vol. 56, no. 8, pp. 21402143, 2009.

[90] E. S. Gang, B. L. Nguyen, Y. Shachar, L. Farkas, L. Farkas, B. Marx, D. Johnson, M. C. Fishbein, C. Gaudio, and S. J. Kim, "Dynamically shaped magnetic fields: initial animal validation of a new remote electrophysiology catheter guidance and control system," Circulation: Arrhtymia and Electrophyisiology, vol. 4, no. 5, pp. 770-777, 2011.

[91] F. P. Gosselin, V. Lalande, and S. Martel, "Characterization of the deflections of a catheter steered using a magnetic resonance imaging system," Am. Assoc. Medical Physics, vol. 38, no. 9, pp. 4994-5002, 2011

[92] A. M. Kim, M. Turakhia, J. Lu, N. Badhwar, B. K. Lee, R. J. Lee, G. M. Marcus, H. Zian, M. Scheinman, and J. E. Olgin, "Impact of remote magnetic catheter navigation on ablation fluoroscopy and procedure time," Pacing and Clinical Electrophysiology, vol. 31, no. 11, pp. 1399 1404, 2008.

[93] M. A. Tavallaei, Y. Thakur, S. Haider, and M. Drangova, "A magneticresonance-imaging-compatible remote catheter navigation system," IEEE Trans. Biomedical Engineering, vol. 60, no. 4, pp. 899-905, 2013.

[94] A. Thiagalingam, A. D' Avila, F. Lori, J. Guerrero, H. Lambert, G. Leo, J. N. Ruskin, and V. Y. Reddy, "Importance of catheter contact force during irrigated radiofrequency ablation: evaluation in porcine ex vivo model using a force-sensing catheter," J. Cardiovascular Electrophysiology, vol. 21, no. 7, pp. 806-811, 2010.

[95] P. Polygerinos, D. Zbyszewski, T. Scaeffter, R. Ravazi, L. D. Seneviratne, and K. Althoefer, "MRI-compatible fiber-optic force sensors for catheterization procedures," IEEE Sensors Journal, vol. 10, no. 10, pp. 1598-1608, 2010.

[96] K. Yokoyama, H. Nakagawa, D. C. Shah, H. Lambert, G. Leo, N. Aeby, A. Ikeda, J. V. Pitha, T. Sharma, R. Lazzara, and W. M. Jackman, "Novel contact force sensor incorporated in irrigated radio frequency ablation catheter predicts lesion size and incidence of steam pop and thrombus," Circulation: Arrhtymia and Electrophyisiology, vol. 1, no. 5, pp. 354-362, 2008 .

[97] P. E. Dupont, J. Lock, B. Itkowitz, and E. Butler, "Design and control of concentric-tube robots," IEEE Trans. Robotics, vol. 26, no. 2, pp. 209-225, 2010.

[98] R. J. Webster III, J. M. Romano, and N. J. Cowan, "Mechanics of precurved-tube continuum robots," IEEE Trans. Robotics, vol. 25, no. 1, pp. 67-78, 2009.

[99] J. Burgner, P. J. Swaney, D. C. Rucker, H. B. Gilbert, P. R. Russel III, K. D. Weaver, and R. J. Webster III, "A bimanual teleoperated system for endonasal skull base surgery," IEEE/RSJ Int. Conf. Intelligent Robots and Systems, pp. 2517-2523, 2011.

[100] A. Gosline, N. V. Vasilyev, E. Butler, C. Folk, A. Cohen, R. Chen, N. Lang, P. J. del Nido, and P. E. Dupont, "Percutaneous intracardiac beating-heart surgery using metal MEMS tissue approximation tools," Int. J. Robotics Research, vol. 31, no. 9, pp. 1081-1093, 2012.

[101] Y.-L. Park, S. Elayaperumal, B. Daniel, S. C. Ryu, M. Shin, J. Savall, R. J. Black, B. Moslehi, and M. R. Cutkosky, "Real-time estimation of 3-d needle shape and deflection for MRI-guided interventions," IEEE/ASME Trans. Mechatronics, vol. 15, no. 6, pp. 906-915, 2010.

[102] R. J. Roesthuis, M. Kemp, J. J. van den Dobbelsteen, and S. Misra, "Three-dimensional needle shape reconstruction using an array of fiber bragg grating sensors," IEEE/ASME Trans. Mechatronics, pp. 1-12, 2013.

[103] H. Su, D. C. Cardona, W. Shang, A. Camilo, G. A. Cole, D. C. Rucker, R. J. Webster III, and G. S. Fischer, "A MRI-guided concentric tube continuum robot with piezoelectric actuation: a feasibility study," IEEE Int. Conf. Robotics and Automation, pp. 1939-1945, 2012.

[104] T. Nakano, N. Sugita, T. Ueta, Y. Tamaki, and M. Mitsuishi, "A parallel robot to assist vitreoretinal surgery," Int. J. Computer Assisted Radiology and Surgery, vol. 4, no. 6, pp. 517-526, 2009.

[105] F. Ulrich, C. Bergeles, J. Pokki, O. Ergeneman, S. Erni, G. Chatzipirpiridis, S. Pané, C. Framme, and B. J. Nelson, "Mobility experiments with microrobots for minimally invasive intraocular surgery," Investigative Ophthalmology and Visual Science, vol. 54, no. 4, pp. 2853-2863, 2013.

[106] R. P. Feynman, "There's plenty of room at the bottom," Engineering and Science, vol. 23, no. 5, pp. 22-36, 1960.

[107] C. Spada, G. Spera, M. Riccioni, L. Biancone, L. Petruzziello, A. Tringali, P. Familiari, M. Marchese, G. Onder, M. Mutignani, V. Perri, C. Petruzziello, F. Pallone, and G. Costamagne, "A novel diagnostic tool for detecting functional patency of the small bowel: the given imaging patency capsule," Endoscopy, vol. 37, no. 9, pp. 793-800, 2005.

[108] P. Glass, E. Cheung, and M. Sitti, "A legged anchoring mechanism for capsule endoscopes using micropatterned adhesives," IEEE Trans. Biomedical Engineering, vol. 55, no. 12, pp. 2759-2767, 2007.

[109] M. Quirini, A. Menciassi, S. Scapellato, C. Stefanini, and P. Dario, "Design and fabrication of a motor legged capsule for the active exploration of the gastrointestinal tract," IEEE/ASME Trans. Mechatronics, vol. 13 , no. 2 , pp. 169-179, 2008.

[110] B. Kim, S. Lee, J. H. Park, and J.-O. Park, "Design and fabrication of a locomotive mechanism for capsule-type endoscopes suing shape memory alloys (SMAs)," IEEE/ASME Trans. Mechatronics, vol. 10, no. 1 , pp. 77-86, 2005.

[111] A. Uehara and K. Hoshina, "Capsule endoscope norika system," Minimally Invasive Therapy and Allied Technologies, vol. 1, pp. 227 234, 2003.

[112] J. Keller, C. Fibbe, F. Volke, J. Gerber, A. C. Mosse, M. ReinmannZawadzki, E. Rabinovitz, P. Layer, and P. Swain, "Remote magnetic control of a wireless capsule endoscope in the esophagus is safe and feasible: results of a randomized, clinical trial in healthy volunteers," Gastrointestinal Endoscopy, vol. 72, no. 5, pp. 941-946, 2012.

[113] G. Kósa, P. Jakab, G. Székely, and N. Hata, "MRI driven magnetic microswimmers," J. Biomedical Microdevices, vol. 14, pp. 165-178, 2012

[114] F. Carpi, N. Kastelein, M. Talcott, and C. Pappone, "Magnetically controllable gastrointestinal steering of video capsules," IEEE Trans. Biomedical Engineering, vol. 58, no. 8, pp. 231-234, 2011.

[115] A. W. Mahoney and J. J. Abbott, "Managing magnetic force applied to a magnetic device by a rotating dipole field," Applied Physics Letters, vol. 99, no. 134103, pp. 1-3, 2011

[116] G. Ciuti, P. Valdastri, A. Menciassi, and P. Dario, "Robotic magnetic steering and locomotion of capsule endoscope for diagnostic and surgical endoluminal procedures," Robotica, vol. 28 , no. 2, pp. 199207, 2010.

[117] E. Gultepe, J. S. Randhawa, S. Kadam, S. Yamanaka, F. M. Selaru, E. J. Shin, A. N. Kalloo, and D. H. Gracias, "Biopsy with thermallyresponsive untethered microtools," Advanced Materials, vol. 25, no. 4, pp. 514-519, 2013.

[118] J. L. Gorlewicz, S. Battaglia, B. F. Smith, G. Ciuti, J. Gerding, A. Menciassi, K. L. Obstein, P. Valdastri, and R. J. Webster III, "Wireless insufflation of the gastrointestinal tract," IEEE Trans. Biomedical Engineering, vol. 60, no. 5, pp. 1225-1233, 2013.

[119] M. Salerno, G. Ciuti, G. Lucarini, R. Rizzo, P. Valdastri, A. Menciassi, A. Landi, and P. Dario, "A discrete-time localization method for capsule 
endoscopy based on on-board magnetic sensing," Measurement Science and Technology, vol. 23, no. 015701, pp. 1-10, 2012.

[120] K. M. Popek, A. W. Mahoney, and J. J. Abbott, "Localization method for a magnetic capsule endoscope propelled by a rotating magnetic dipole field," IEEE Int. Conf. Robotics and Automation, pp. 5328-5333, 2013.

[121] S. Tognarelli, V. Castelli, G. Ciuti, C. Di Natali, E. Sinibaldi, P. Dario, and A. Menciassi, "Magnetic propulsion and ultrasound tracking of endovascular devices," J. Robotic Surgery, vol. 6, no. 1, pp. 5-12, 2012.

[122] M. Sendoh, K. Ishiyama, and K. I. Arai, "Direction and individual control of magnetic micromachine," IEEE Trans. Magnetics, vol. 38, no. 5, pp. 3356-3358, 2002.

[123] N. D. Nelson, J. Delacenserie, and J. J. Abbott, "An empirical study of the role of magnetic, geometric, and tissue properties on the turning radius of magnetically driven screws," IEEE Int. Conf. Robotics and Automation, pp. 5352-5357, 2013.

[124] S. Martel, O. Felfoul, J. Mathieu, A. Chanu, S. Tamaz, M. Mohammadi, M. Mankiewicz, and N. Tabatabaei, "MRI-based medical nanorobotic platform for the control of magnetic nanoparticles and flagellated bacteria for target interventions in human capillaries," Int. J. Robotics Research, vol. 28, no. 9, pp. 1169-1182, 2009.

[125] C. Bergeles, M. P. Kummer, B. E. Kratochvil, C. Framme, and B. J. Nelson, "Steerable intravitreal inserts for drug delivery: in vitro and ex vivo mobility experiments," Int. Conf. Medical Image Computing and Computer Assisted Intervention, pp. 33-40, 2011.

[126] S. N. Tabatabaei, S. Duchemin, H. Girouard, and S. Martel, "Towards MR-navigable nanorobotic carriers for drug delivery into the brain," IEEE Int. Conf. Robotics and Automation, pp. 727-732, 2012.

[127] S. Martel, J.-B. Mathieu, O. Felfoul, A. Chanu, E. Aboussouan, S. Tamaz, P. Pouponneau, L. Yahia, G. Beaudoin, G. Soulez, and M. Mankiewicz, "Automatic navigation of an untethered device in the artery of a living animal using a conventional clinical magnetic resonance imaging system," Applied Physics Letters, vol. 90, p. 114105, 2007

[128] S. Park, K. Cha, and J. Park, "Development of biomedical microrobot for intravascular therapy," Int. J. Advanced Robotic Systems, vol. 7, no. 1, pp. 91-98, 2010.

[129] B. R. Donald, C. G. Levey, C. D. McGray, I. Paprotny, and D. Rus, "An untethered, electrostatic, globally controllable MEMS micro-robot," IEEE J. Microelectromechanical Systems, vol. 15, no. 1, pp. 1-15, 2006.

[130] M. S. Sakar, E. B. Steager, D. H. Kim, K. Agung, M. Kim, V. Kumar, and G. Pappas, "Modeling, control and experimental characterization of microbiorobots," Int. J. Robotics Research, vol. 30, no. 6, pp. 647658, 2011.

[131] S. Martel, M. Mohammadi, O. Felfoul, Z. Lu, and P. Pouponneau, "Flagellated magnetotactic bacteria as controlled MRI-trackable propulsion and steering systems for medical nanorobots operating in the human microvasculature," Int. J. Robotics Research, vol. 28, no. 4, pp. 571-582, 2009.

[132] B. Behkam and M. Sitti, "Design methodology for biomimetic propulsion of miniature swimming robots," J. Dynamical Systems, Measurement and Control, vol. 128, pp. 36-43, 2006

[133] W. Hu, K. S. Ishii, Q. Fan, and A. Ohta, "Hidrogel microrobots aactuated by optically generated vapour bubbles," Lab on a Chip, vol. 12, no. 19, pp. 3821-3826, 2012.

[134] J. J. Abbott, K. E. Peyer, M. C. Lagomarsino, L. Zhang, L. Dong, I. K. Kaliakatsos, and B. J. Nelson, "How should microrobots swim?" Int. J. Robotics Research, vol. 28, no. 11-12, pp. 1434-1447, 2009.

[135] C. Bergeles, B. E. Kratochvil, and B. J. Nelson, "Visually servoing magnetic intraocular microdevices," IEEE Trans. Robotics, vol. 28, no. 4, pp. 798- 809, 2012.

[136] T. N. Z. Herald. (2013, July) Kiwi designer creates 3d-printed exoskeleton for broken bones. [Online]. Available: http://www.nzherald. co.nz/technology/news/article.cfm?c_id=5\&objectid=10894990

[137] C. Osborne. (2013, January) "Magic Arms" 3D printed exoskeleton nominated for design award. [Online]. Available: http://www.smartplanet.com/blog/bulletin/ -8216magic-arms-3d-printed-exoskeleton-nominated-for-design-award/ 11238

[138] M. Salerno, S. Tognarelli, C. Quaglia, P. Dario, and A. Menciassi, "Anchoring frame for intra-abdominal surgery," Int. J. Robotics Research, vol. 32, no. 3, pp. 360-370, 2013.

[139] L. Ricotti and A. Menciassi, "Engineering stem cells for future medicine," IEEE Trans. Biomedical Engineering, vol. 60, no. 3, pp. 727-734, 2013.
[140] M. K. Llewellyn, P. Dario, A. Menciassi, and E. Sinibaldi, "Magnetic dragging of vascular obstructions by means of electrostatic and antibody binding," IEEE Int. Conf. Robotics and Automation, pp. 25042509, 2012.

[141] H. Marcus, D. Nandi, A. Darzi, and G.-Z. Yang, "Surgical robotics through a keyhole: from today's translational barriers to tomorrow's "disappearing" robots," IEEE Trans. Biomedical Engineering, vol. 60 , no. 3, pp. 674-681, 2013.

[142] M. Wadman, "Science agencies prepare for cuts," Nature, vol. 494, pp. 158-159, 2013.

[143] A. Abbott, "Europe's leaders slash proposed research budget," Nature, 2013.

[144] N. Ghaffarzadegan, R. C. Larson, and J. Hawley, "The coming hangover: magnified effects of sequestration on research enterprises," MIT Engineering Systems Division Working Paper Series, vol. 03, pp. 1-3, 2013.

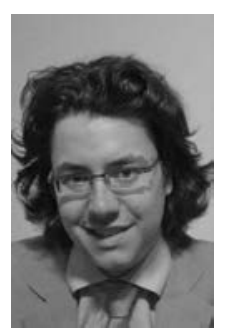

Christos Bergeles (M'11) received the Ph.D. degree in mechanical engineering from ETH Zurich, Switzerland, in 2011.

He is currently a Hamlyn Fellow with the Hamlyn Centre for Robotic Surgery, Imperial College London. His main research interests include miniaturized telesurgical robotic platforms and medical image processing for robot guidance.

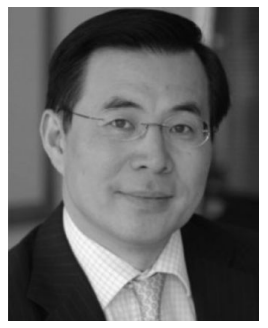

Guang-Zhong Yang (F'11) received the Ph.D. degree in computer science from Imperial College London, London, U.K.

$\mathrm{He}$ is the Director, Cofounder, and currently chairs the Hamlyn Centre for Robotic Surgery, Imperial College London. His main research interests include biomedical imaging, sensing, and robotics.

Dr. Yang is a Fellow of the Royal Academy of Engineering, the Institution of Engineering and Technology and the American Institute for Medical Biological Engineering. 